\title{
A 100kS/s CMOS Time-Domain Temperature Sensor for Wireless Sensor Networks
}

\author{
Yuanxin Bao, Wenyuan $\mathrm{Li}^{+}$and Jun Feng \\ Institute of RF-\&OE-ICs, Southeast University, Nanjing 210096, China
}

\begin{abstract}
This paper presents a smart temperature sensor with digital output, which is suitable to be used in temperature monitoring of wireless sensor networks. A ring oscillator and bias voltage is designed as the temperature sensor to generate quasi-linear temperature dependent output pulses. One-point calibration and low drop-out regulators is adopted to effectively relieve the impact of process and supply voltage variation. A frequency-to-digital converter (FDC) is used to translate frequency into a digital output code. The sensor is designed in a TSMC $0.18 \mu \mathrm{m} 1 \mathrm{P} 6 \mathrm{M}$ standard CMOS technology and consumes $4 \mathrm{~nJ}$ of energy at conversion rate of $100 \mathrm{kS} / \mathrm{s}$ with a resolution of $0.25^{\circ} \mathrm{C} / \mathrm{LSB}$ and a core area of $0.0275 \mathrm{~mm}^{2}$. The proposed sensor is capable of working in the temperature range of $-45^{\circ} \mathrm{C} \sim 85^{\circ} \mathrm{C}$ with a supply voltage of $1.4 \mathrm{~V} \sim 2.4 \mathrm{~V}$. With the piecewise linear fitting and one-point calibration adopted, the sensor shows an inaccuracy of $-1.2^{\circ} \mathrm{C} \sim 1.2^{\circ} \mathrm{C}$.
\end{abstract}

Keywords: time-domain temperature sensor, wireless sensor networks, low power, wide supply voltage.

\section{Introduction}

Temperature sensor plays an important role in many applications and systems, such as biomedical applications, wireless sensor networks (WSN) and so on. Typically, WSN includes many temperature sensor nodes whose lifetime should be as long as possible. Therefore, small-area, low power consumption and high speed temperature sensors are required for the wireless temperature measuring sensor network.

The temperature sensors are divided into two categories, namely voltage-domain and time-domain temperature sensor. In [1], a voltage-domain temperature sensor was reported which achieves an inaccuracy of $\pm 0.1^{\circ} \mathrm{C}$ over $-55^{\circ} \mathrm{C} \sim 125^{\circ} \mathrm{C}$ temperature range after one-point calibration. However, the chip area and energy per conversion were as high as $4.5 \mathrm{~mm}^{2}$ and $18.75 \mu \mathrm{J}$, respectively. With small chip area and low power consumption, time-domain temperature sensor is better suited for WSN than voltage-domain. In this sensor, frequency, period or duty-cycle is used to express thermal information [2].

In this paper, we present a time-domain, MOSFET-based temperature sensor useful in the temperature range of $-45^{\circ} \mathrm{C} \sim 85^{\circ} \mathrm{C}$, which integrates a bandgap, a low drop-out regulator (LDO), a ring oscillator, a level shifter and an FDC. It achieved an inaccuracy of $-1.2^{\circ} \mathrm{C} \sim 1.2^{\circ} \mathrm{C}$ over the temperature range of $-45^{\circ} \mathrm{C} \sim 85^{\circ} \mathrm{C}$.

\section{Architecture and Operation Principle}

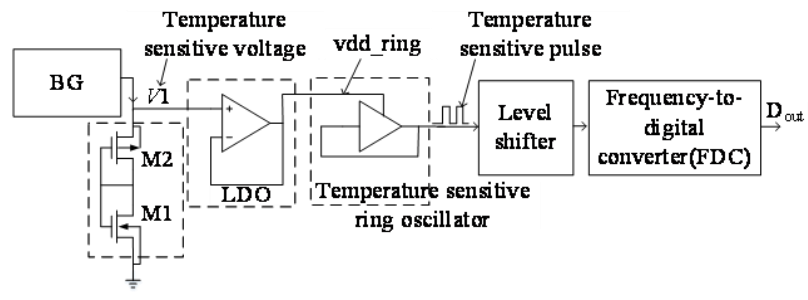

(a)

Fig. 1: (a) Block diagram of the proposed temperature sensor

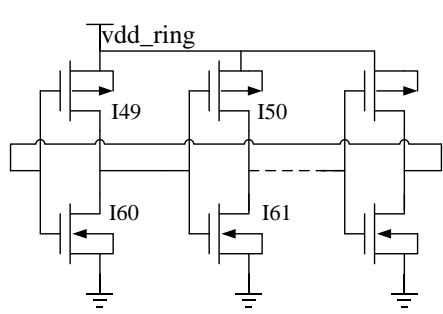

(b)

(b) Schematic of ring oscillator.

\footnotetext{
+ Corresponding author. Tel.: + 86-258-3793303-8109; fax: +86-25-63792882.

E-mail address: 1wy555@seu.edu.cn.
} 
Fig. 1(a) shows the block diagram of the proposed time-domain temperature sensor with one-point calibration and piecewise linear fitting for curvature correction. Temperature is measured by temperature sensitive voltage $(V 1)$ and temperature sensitive ring oscillator. The LDO is used to resolve problem that measurement accuracy is affected by the supply voltage variation. Level shifter is the bridge where different voltage regions transform signal. An FDC is used to digitize the number of pulses for output coding.

In Fig. 1(a), M1 and M2 are operating in saturate region. Hence, the voltage (V1) can be calculated as

$$
V 1=v d d_{-} \text {ring }=\sqrt{2 \cdot \cdot \cdot L_{2} / W_{2} \cdot \mu \cdot c_{o x}}+V_{t h p}+\sqrt{2 \cdot \cdot \cdot L_{1} / W_{1} \cdot \mu \cdot c_{o x}}+V_{t h n},
$$

where temperature insensitive current $(I)$ is generated by the bandgap circuit. The schematic of ring oscillator is depicted in Fig. 1(b). The frequency of the pulse can be calculated as [3]

$$
f=\frac{1}{n \cdot t}=\frac{W}{n \cdot L} \cdot \frac{C_{o x} \mu}{C} \cdot \frac{v d d_{-} \text {ring } \cdot\left(1-V_{t h} / v d d_{-} \text {ring }\right)}{\ln \left(3-4 V_{t h} / v d d_{\_} \text {ring }\right)},
$$

where $\mathrm{C}$ is equivalent capacitance of output node; $\mathrm{n}$ is the number of inverter; $\mathrm{C}_{\mathrm{ox}}$ is the gate oxide capacitance per unit area. TSMC CMOS technology allows us to assume threshold voltage $\left|V_{t h p}\right|=V_{t h n}=V_{t h}$. Substituting Eq.(1) into Eq. (2) acquires the simultaneous solutions as follows:

$$
f=\frac{W}{n \cdot L} \cdot \frac{C_{o x} \mu}{C} \cdot \frac{\left(\sqrt{2 \cdot I \cdot L_{1} / W_{1} \cdot \mu \cdot c_{o x}}+V_{t h}+\sqrt{2 \cdot I \cdot L_{2} / W_{2} \cdot \mu \cdot c_{o x}}\right)}{\ln \left(3-4 V_{t h}\left(\sqrt{2 \cdot I \cdot L_{1} / W_{1} \cdot \mu \cdot c_{o x}}+2 V_{t h}+\sqrt{2 \cdot \cdot \cdot \cdot L_{2} / W_{2} \cdot \mu \cdot \cdot c_{o x}}\right)\right.},
$$

where $\mu(T)=\mu\left(T_{0}\right)\left[T / T_{0}\right]^{-k_{1}}, k_{1}=1.5 \sim 2, V_{t}(T)=V_{t}\left(T_{0}\right)-k_{2}\left(T-T_{0}\right), k_{2}=0.5 \sim 4 m V / K$.

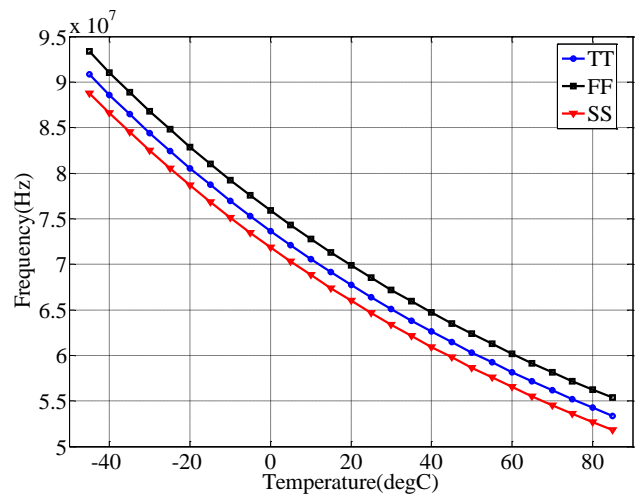

(a)

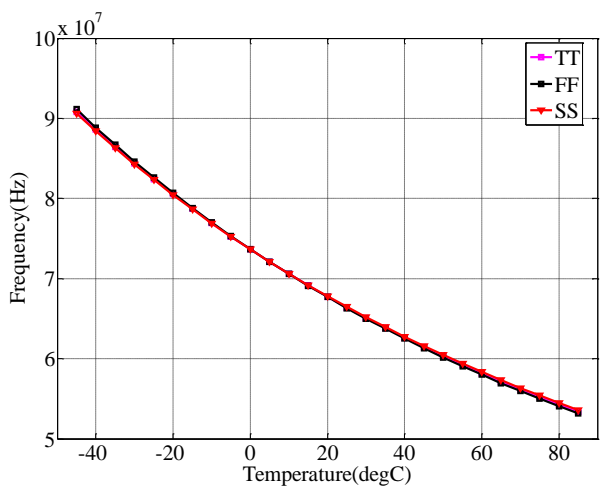

(b)

Fig. 2: (a) Output frequency vs. temperature for different process corner (b) calibrated frequency.

According to Eq.(3), the frequency has negative thermal coefficient. Fig. 2(a) shows the output frequency of temperature sensor versus temperature in different process corners. Fig. 2(b) shows the calibrated frequency in various corners by adding one point calibration. According to corner simulation, onepoint calibration was done at a specific temperature, which almost removed the impact of the process. The frequency range of $53.37 \mathrm{MHz} \sim 90 \mathrm{MHz}$, which ensures the fine resolution at conversion rate of $100 \mathrm{kS} / \mathrm{s}$.

\section{Circuit Details}

\subsection{CMOS bandgap}

CMOS Bandgap circuit is shown in Fig. 3. The PMOS transistor M1, M2 and M3 form current mirrors whose dimensions are the same. The resistance of R1 and R3 is the same. The emitter area of Q2 is N times that of Q1. The current of R1 (R3) can be calculated as

$$
I_{2}=I_{R 1}=I_{R 3}=\frac{V_{A}}{R 1}=\frac{V_{B}}{R 3}=\frac{V_{e b 1}}{R 1}=\frac{V_{e b 1}}{R 3}
$$

The current of R2 (Q1) can be calculated as

$$
I_{1}=I_{R 2}=I_{Q 1}=\frac{V_{e b 1}-V_{e b 2}}{R 2}=\frac{V_{T} \ln N}{R 2}
$$


The output of current equals

$$
I_{M 1}=I_{M 2}=I_{M 3}=I_{1}+I_{2}=\left(\frac{V_{e b 1}}{R 3}+\frac{V_{T} \ln N}{R 2}\right)
$$

where, $V_{e b 1}$ has a temperature coefficient of $-2 \mathrm{mV} /{ }^{\circ} \mathrm{C}, V_{T}$ has a temperature coefficient of $0.086 \mathrm{mV} /{ }^{\circ} \mathrm{C}$.

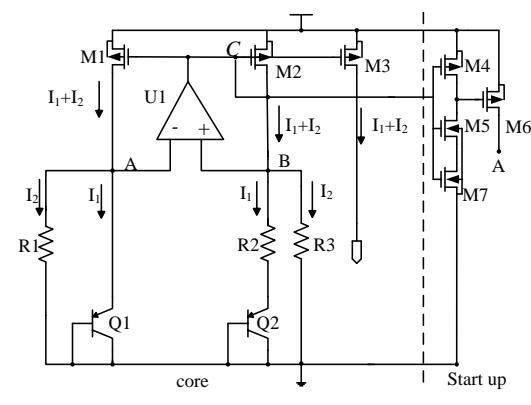

Fig. 3: Schematic of CMOS Bandgap reference.

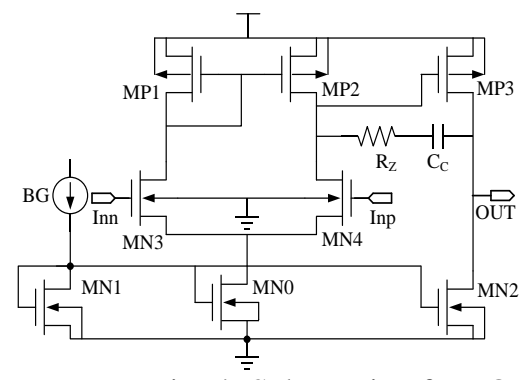

Fig. 4: Schematic of LDO.

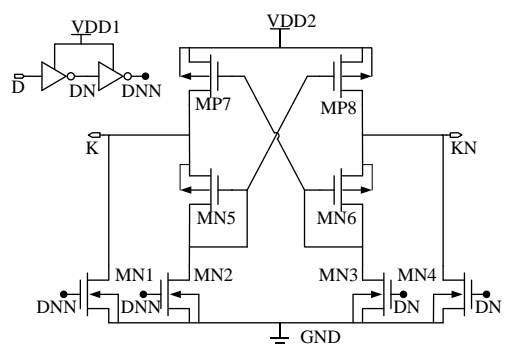

Fig. 5: Schematic of level shifter.

\subsection{Low drop-out regulators (LDO)}

LDO is used to decrease the temperature sensor's sensitivity to supply voltage, which is shown in Fig. 4. The error amplifier with miller compensation is used to achieve stability. MP3 is common source stage and pass device simultaneously, which is used to provide voltage gain and sufficient voltage headroom.

\subsection{Level shifter}

Because ring oscillator and following circuit run at different voltage regions, level shifter is required to convert the logic signal from one voltage region to the other. The level shifter is shown as Fig.5. When D is at GND, MN1 and MN2 turn off, MN3 and MN4 turn on. MN6 and MP7 would be turned on, so that node K is rapidly pulled up to VDD2. The case reverses if D is at VDD1.

\subsection{Frequency-to-digital converter (FDC)}

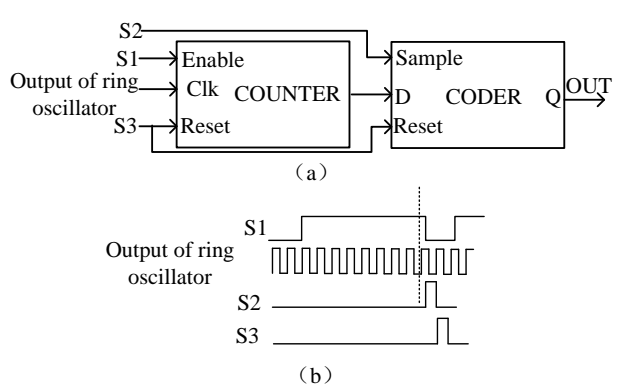

Fig. 6: (a) Block diagram of FDC (b) Time diagram of FDC

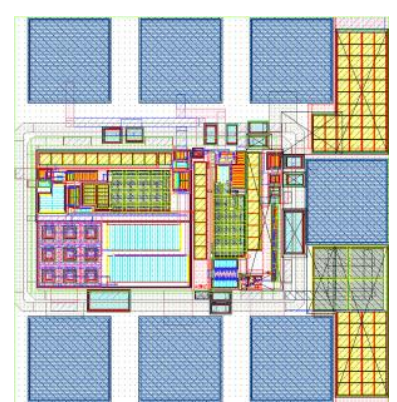

Fig. 7: Layout of the proposed temperature sensor.

The block diagram of FDC is shown as Fig. 6(a), which consists of an asynchronous counter and a coder. The coder adopts piecewise linear fitting technology and one-point calibration. Fig.6(b) shows the time diagram of the FDC. When S1 is high, the counter begins to count the rising edges of the pulse. When S1 is low, the counter stops working. The coder converts output of counter to temperature when S2 is high. For the next measurement, $\mathrm{S} 3$ resets the counter and coder.

\section{Simulation Results}

The proposed time-domain temperature sensor has been designed in a TSMC $0.18 \mu \mathrm{m}$ CMOS technology. Fig. 7 shows the layout of sensor, and the core circuit area is $0.0275 \mathrm{~mm}^{2}$. All simulations were done in the temperature range of $-45^{\circ} \mathrm{C} \sim 85^{\circ} \mathrm{C}$. The simulated temperature inaccuracy is shown in Fig.8(a) for different supply voltages. The inaccuracy are within $-1.2^{\circ} \mathrm{C} \sim 1.2^{\circ} \mathrm{C}$. For supply voltage variations, the temperature sensor shows a supply voltage dependency of $0.01^{\circ} \mathrm{C} / \mathrm{V}$ from $1.4 \mathrm{~V}$ to $2.4 \mathrm{~V}$ with a fine resolution of $0.25^{\circ} \mathrm{C} / \mathrm{LSB}$ in simulation. Histogram of temperature inaccuracy at $-25^{\circ} \mathrm{C}$ is shown in Fig. $8(\mathrm{~b})$. Monte Carlo simulations show that the proposed sensor has dinky mismatch dependency, ensuring good yield. 


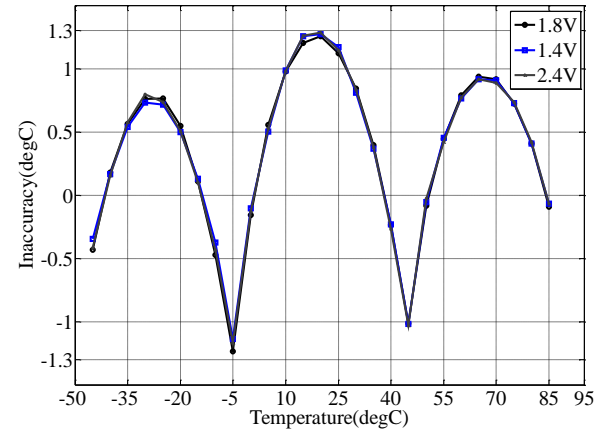

(a)

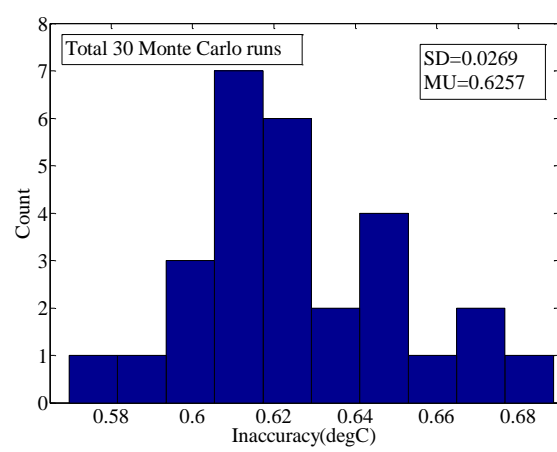

(b)

Fig. 8: (a) Temperature inaccuracy for different supply voltages (b) Histogram of temperature inaccuracy at $-25^{\circ} \mathrm{C}$.

Table 1. Performance Comparison with State-of-the-Art Temperature Sensors

\begin{tabular}{|c|c|c|c|}
\hline Parameter & This work & {$[4]$} & {$[5]$} \\
\hline Technology $(\mu \mathrm{m})$ & 0.18 CMOS & 0.18 CMOS & 0.13 CMOS \\
\hline Inaccuracy $\left({ }^{\circ} \mathrm{C}\right)$ & $-1.2 \sim 1.2$ & $-0.1 \sim 1$ & $-1.7 \sim 1.5$ \\
\hline Supply voltage $(\mathrm{V})$ & $1.4 \sim 2.4$ & 0.9 & $0.2 \sim 0.5$ \\
\hline Temperature $\left({ }^{\circ} \mathrm{C}\right)$ & $-45 \sim 85$ & $-55 \sim 125$ & $0 \sim 100$ \\
\hline Conversion Rate $($ Sample/sec) & $100 \mathrm{k}$ & 20 & 10 \\
\hline
\end{tabular}

Table 1 compares the main performances of proposed temperature sensor with state-of-the-art temperature sensor in [4, 5]. It can be learned from the table that the proposed sensor is able to work at widest supply voltage range. The conversion rate of the proposed temperature sensor is highest.

\section{Conclusion}

With the help of LDO, the proposed sensor operates from a $1.4 \mathrm{~V} \sim 2.4 \mathrm{~V}$ supply voltage with accuracy variation of $0.01^{\circ} \mathrm{C} / \mathrm{V}$. After applying one-point calibration over a temperature range of $-45^{\circ} \mathrm{C} \sim 85^{\circ} \mathrm{C}$, the proposed sensor still achieves an inaccuracy $-1.2^{\circ} \mathrm{C} \sim 1.2^{\circ} \mathrm{C}$ with a piecewise linear fitting. The energy consumption is measured to be $4 \mathrm{~nJ} /$ conversion under $1.8 \mathrm{~V}$ supply voltage. With the present simulation performance the proposed sensor is suitable for temperature monitoring of wireless sensor networks.

\section{Acknowledgements}

We are sincerely thankful for the support from the Project Funded by the Priority Academic Program Development of Jiangsu Higher Education Institutions (PAPD, No.1104007003), Natural Science Foundation of China (No.61471119) and Topnotch Academic Programs Project of Jiangsu Higher Education Institutions (TAPP) PPZY2015A035.

\section{References}

[1] M. A. P. Pertijs, K. A. A. Makinwa and J. H. Huijsing, "A CMOS smart temperature sensor with a $3 \sigma$ inaccuracy of $\pm 0.1^{\circ} \mathrm{C}$ from $-55^{\circ} \mathrm{C}$ to $125^{\circ} \mathrm{C}$," Solid-State Circuits, IEEE Journal of, vol. 40, pp. 2805-2815, 2005.

[2] P. Chen, Y. Hu, J. Liou, and B. Ren, "A 486kS/s CMOS time-domain smart temperature sensor with $0.85^{\circ} \mathrm{C} / 0.78^{\circ} \mathrm{C}$ voltage-calibrated error," in Circuits and Systems (ISCAS), 2015 IEEE International Symposium on Lisbon, Portugal: IEEE, 2015, pp. 2109-2112.

[3] D. Ha, K. Woo, S. Meninger, T. Xanthopoulos, E. Crain, and D. Ham, "Time-Domain CMOS Temperature Sensors With Dual Delay-Locked Loops for Microprocessor Thermal Monitoring," Very Large Scale Integration (VLSI) Systems, IEEE Transactions on, vol. 20, pp. 1590-1601, 2011.

[4] B. Wang, M. K. Law, and F. Tang, "A sub-1V BJT-based CMOS temperature sensor from $-55^{\circ} \mathrm{C}$ to $125^{\circ} \mathrm{C}$," in Circuits and Systems (ISCAS), 2012 IEEE International Symposium on Seoul: IEEE, 2012, pp. 3114-3117.

[5] D. A. Kamakshi, A. Shrivastava and B. H. Calhoun, "A $23 \mathrm{nW}$ CMOS ultra-low power temperature sensor operational from $0.2 \mathrm{~V}$," in SOI-3D-Subthreshold Microelectronics Technology Unified Conference (S3S), 2015 IEEE Rohnert Park, CA: IEEE, 2015, pp. 1-3. 